\title{
DESARROLLO SOSTENIBLE PARA LA EDUCACIÓN EN DISEÑO
}

Puentes-Bedoya, Janeth

DESARROLLO SOSTENIBLE PARA LA EDUCACIÓN EN DISEÑO

PANORAMA, vol. 14, núm. 26, 2020

Politécnico Grancolombiano, Colombia

Disponible en: http://www.redalyc.org/articulo.oa?id=343963784001

DOI: https://doi.org/10.15765/pnrm.v14i26.1479

Esta obra está bajo una Licencia Creative Commons Atribución-NoComercial-SinDerivar 4.0 Internacional. 
Artículos de investigación científica y tecnológica

DESARROLLO SOSTENIBLE PARA
LA EDUCACIÓN EN DISEÑO

SUSTAINABLE DEVELOPMENT FOR DESIGN

EDUCATION

DESENVOLVIMENTO SUSTENTÁVEL PARA A

EDUCAÇÃO EM DESIGN

\author{
Janeth Puentes-Bedoya jpuentesb@poligran.edu.co \\ Institución Universitaria Politécnico Grancolombiano, Colombia
}

PANORAMA, vol. 14, núm. 26, 2020

Politécnico Grancolombiano, Colombia

Recepción: 26 Julio 2018

Aprobación: 26 Septiembre 2019

DOI: https://doi.org/10.15765/ pnrm.v14i26.1479

Redalyc: http://www.redalyc.org/ articulo.oa?id=343963784001

\section{BY-NC-ND}

Resumen: El cambio en los valores, actitudes y patrones de consumo de los seres humanos ha afectado severamente los territorios que tradicionalmente ocupaban un espacio sostenible en la sociedad, siendo el cuidado y la educación un espacio fundamental para la conservación.

Las competencias académicas de los diseñadores tienen como objetivo, además del espacio teórico, el desarrollo de habilidades proyectuales y aprendizaje en el actuar propio de la disciplina, razón por la que educación en diseño para la sostenibilidad implica identificar y formular problemas descritos desde las bases ambientales y disciplinares, incursionando en áreas transversales y en temáticas sostenibles aplicadas al proyecto formativo propuesto, aunado a la inclusión de elementos que permitan incorporar las realidades laborales para escenarios futuros.

Palabras clave: Diseño sostenible, educación, diseño industrial, laboratorios de diseño, ecodiseño.

Abstract: The change in the values, attitudes and consumption patterns of human beings has severely affected the territories that traditionally occupy a sustainable space in society, being care and education a fundamental space for conservation.

In addition to the theoretical space, the academic competencies of the designers have as their objective the development of project skills and learning in the discipline's own act, which is why education in design for sustainability implies identifying and formulating related problems from the grassroots environmental and disciplinary, venturing into cross-cutting areas and sustainable themes applied to the proposed training project, coupled with the inclusion of elements that include incorporating labor realities for future scenarios.

Keywords: Sustainable design, education, industrial design, design labs, eco design.

Resumo: A alteração dos valores, atitudes e padrões de consumo dos seres humanos afectou gravemente os territórios que tradicionalmente ocupavam um espaço sustentável na sociedade, ser o cuidado e a educação um espaço fundamental para a conservação. Para além do espaço teórico, as competências académicas dos designers visam desenvolver competências de projecto e aprender a agir dentro da disciplina. Por este motivo, a educação em design para a sustentabilidade implica identificar e formular problemas descritos a partir de bases ambientais e disciplinares, entrando em áreas transversais e temas sustentáveis aplicados ao projecto de formação proposto, juntamente com a inclusão de elementos que permitam a incorporação de realidades laborais para cenários futuros.

Palavras-chave: Design sustentável, educação, desenho industrial, laboratórios de design, eco design. 


\section{INTRODUCCIÓN}

Los países buscan comprometerse con el desarrollo sostenible, enmarcado en lo definido por la Comisión mundial del medio ambiente: «... como la satisfacción de las necesidades de la generación presente sin comprometer la capacidad de las generaciones futuras para satisfacer sus propias necesidades» (informe titulado «Nuestro futuro común», de 1987, Comisión Mundial sobre el Medio Ambiente y el Desarrollo); complementado por la agenda de los Objetivos de Desarrollo Sostenible, ODS 2030, en el apartado 4.7, que incluye la Educación para el desarrollo sostenible y la ciudadanía mundial (UNESCO, 2012, p. 9).

Es clara la visión de la UNESCO para el 2030 respecto a los ODS (objetivos del desarrollo sostenible) relacionados con la educación, por esto, incluye un espacio relevante a la Educación y la ciudadanía mundial para lograr el desarrollo sostenible. Confluyen allí aspectos holísticos de transformación e inclusión social y natural. La educación debe ser un proyecto global enfocado a cumplir estos diecisiete objetivos y aunar fuerzas para combatir la pobreza, de tal manera que todos los esfuerzos estarán enfocados al desarrollo y la educación, basados en propuestas contemporáneas de Pedagogía y Educación.

La educación para el desarrollo sostenible busca crear un mejor mañana para todos, siendo la educación el factor principal para lograr la equidad, la paz y la igualdad. Las mejoras al respecto a nivel mundial deben incluir evaluación de los estados y deficiencias, y cómo cuantificar el desarrollo para que este sea global y permita acoger a la mayor cantidad de personas. En el ámbito de la formación en diseño para la sostenibilidad se incluyen los diferentes elementos de los planes de estudio que pueden contribuir a un futuro sostenible (UNESCO, 2012, p. 8).

Desde la academia, estos factores deben interiorizarse. Es necesario que la disciplina del diseño asuma la responsabilidad y construya un espacio de diseño para la sostenibilidad, que permee los escenarios educativos en Latinoamérica -fundamentados en entornos desactualizados y vetustosdonde los componentes, las formas y las funciones se generan desde la producción industrial basada en economías tradicionales que favorecen las industrias del petróleo con el suministro de crudo como materia prima desde hace décadas.

Es así como todo crecimiento exponencial llega necesariamente a un punto en el que la oferta excede la capacidad de demanda genuina o forzada. La mayoría de las veces, ese punto llega antes que otro todavía más dramático: el momento en el que se alcanza el límite natural del abastecimiento (Bauman, 2013, p. 61).

Es importante tener en cuenta que los productos industriales ejercen un impacto importante en la tierra, derivado de los procesos de producción, los cuales se gestionan ambientalmente desde el ACV (Análisis de Ciclo de Vida del producto) (Ahmad, Wong, Tseng, \& Wong, 2018; Reuter \& Reiter, 2019); lo que permite a los diseñadores vislumbrar en cada una de las fases de construcción cómo se afectará el medio ambiente, pero sobre todo cómo se logran elaborar, implementar y evaluar proyectos 
que mejoren las condiciones de materialización desde propuestas más amigables con el medio ambiente (Wiprächtiger, Haupt, Heeren, Waser, $\&$ Hellweg, 2020). La sostenibilidad aunada al diseño puede preparar los procesos asociados a la selección adecuada de materiales, reutilización de agua, disminución de energía y optimización de los elementos asociados a la industrialización y producción, que merecen transformarse en un diferencial para la disciplina y ampliar el ámbito epistémico del diseño para la sostenibilidad (Tsitman \& Proshunina, 2019). Sin dejar de lado las propuestas de servicios compartidos, alquiler y reparación como alternativa a la compra, venta y desecho. Medir el impacto del diseño para la sostenibilidad en la sociedad será posible cuando las necesidades y los productos afecten positivamente en los resultados del ejercicio proyectual y de producto, es decir, cuando el diseñador interiorice los conceptos de la sostenibilidad y brinde alternativas desde una visión colectiva del uso de los objetos.

Josep María Galip (2014), citado por Niño (2015, p. 106), aseguró que la obsolescencia programada reenvía en su aceptación más epidérmica unos "malos": los fabricantes y sus malévolos ingenieros y diseñadores industriales, que planean de forma consciente productos que se estropean tan rápidamente como lo permite el mercado para provocar nuevas ventas a través de innovaciones poco relevantes sino engañosas (Kinokuni, Ohori, \& Tomoda, 2019; Paricio, Peña, \& Miralbes, 2019).

Las dinámicas sociales y culturales que acompañan y modifican los procesos educativos en diseño fortalecerán los aspectos deficientes en los que la disciplina ha recaído, debido a las permanentes modificaciones de forma que no permiten la creación de espacios contemporáneos de educación en diseño y creación de una pedagogía alternativa, que corresponda a dinámicas culturales, sociales y tecnológicas actuales de los estudiantes de diseño. En palabras de Papanek (1997): “[...] el diseño, la herramienta de modelado más poderosa que hasta ahora ha sido utilizada por el hombre para moldearse, ir a su entorno, seguir adelante" (p. 229). En otras investigaciones, más recientes, se da un ejemplo de la estructura y las tareas del sistema de diseño y gestión del desarrollo sostenible, así como de las posibilidades de su aplicación como sistema de aprendizaje electrónico en el ámbito del diseño y la gestión del desarrollo sostenible (Bolshakov, Shamaeva, \& Popov, 2019; Lai \& Peng, 2020).

Aprehendiendo del universo que exhibe un extraordinario conjunto de principios fundamentales que jamás se contradicen y siempre están en concordancia, mostrando algunas de estas concordancias o adaptaciones elevados niveles exponenciales de rarezas cinegéticas, algunas de las cuales suponen interacciones energéticas a niveles geométricos de cuarta potencia (Papanek, 1977, p. 10), tambien se analizan los costos/beneficios económicos y sociales de las estrategias de adaptación de los diseños arquitectonicos en la actualidad y el diseño primario de las casas sin barreras dentro del conntexto de la sostenibilidad, teniendo en cuenta los usuarios (Malik \& Mikolajczak, 2019). 


\section{Diseño colombiano}

Las escuelas latinoamericanas tienen influencia de la Bauhaus, y sus docentes y discentes, muchos de ellos ahora norteamericanos, fueron acogidos en la Segunda Guerra Mundial como objetos de fortalecimiento científico, industrial y académico. En el caso colombiano, el diseño industrial traído por Jaime Gutiérrez Lega y Andrés Sicard -quienes fueron discípulos de academias norteamericanas e italianas de arte aplicado- y en su retorno a mediados de los setentas facilitaron la progresión de las escuelas de diseño en la ciudad de Bogotá, con el fin específico de fortalecer la industria y desarrollar un manifiesto de pensamiento alternativo, bien acogido en este momento histórico. Otros estudios proponen un marco del pensamiento de diseño sostenible, en el que se proponen cinco enfoques de pensamiento dinámico: pensar con el cuerpo, pensar con la mente, pensar con el corazón, pensar con las manos y pensar con el alma (Li, Ho, \& Yang, 2019).

Bogotá, en 1930, hace uno de sus mayores esfuerzos para industrializarse; aun así, una de las regiones más desarrolladas es Antioquia, pues la bonanza cafetera influenció el progreso de estas zonas posibilitando la descentralización de la industria, logrando nuevos conglomerados industriales en la geografía nacional; esto también motivó la fundación de escuelas locales de diseño industrial (Bolshakov et al., 2019), como la de la Universidad Pontificia Bolivariana (UPB). Basados en principios de economía, orden y belleza se establece el punto de inspiración para proyectos como equipamiento total de un municipio, una calle, un parque, una sala cinematográfica o una cantina de obreros y aún más: no solo se encargaban de generar nuevos objetos, sino que con ellos lograban diversas funciones, tenían facilidad de montaje y elementos unificadores, estandarizados e intercambiables, permitiendo así una gran cantidad de aplicaciones y combinaciones y logrando una respuesta coherente con un contexto social lleno de penurias y carencias.

Las ideas de estandarización, de normalización en la construcción de elementos que se prestasen a muchas combinaciones, aunque formuladas de modo todavía embrionario, superaban ya medio siglo de las nociones de la época sobre estos problemas, vitales para la producción en serie (Salinas, 1992, p. 115), lo que hace que el diseño universal para personas sea una respuesta eficiente a las necesidades que surgen de nuestra sociedad (Malik \& Mikolajczak, 2019).

Vale la pena mencionar que Colombia carece de una infraestructura industrial que apoye al diseño. La década de los 70 se caracterizó económicamente por impulsar la industria cafetera, teniendo una bonanza coincidencial entre 1970 y 1979, que hizo que descendiera el empleo y el crecimiento industrial en las siguientes décadas. Las industrias del tabaco, calzado, confecciones y bebidas, creadas en decenios anteriores, se vieron desaceleradas; el diseño industrial merecía apoyar los procesos de la regeneración industrial y el valor agregado como motor de las exportaciones y crecimiento económico, aun así su nacimiento surge como una necesidad académica, la inclusión en el contexto colombiano 
y su aplicación, es por esto que sigue teniendo un camino borroso para la industria y los gobiernos, quienes aún desconocen la influencia de la disciplina en el crecimiento y desarrollo del país, incluyendo aspectos asociados a la innovación y la investigación (Teng \& Qu, 2018; Yi-Fei, 2017).

Los años 90 fueron un momento importante para la industria colombiana. La productividad laboral era similar a la de Estados Unidos, lo que debió haberse aprovechado para la creación de empresas y fortalecimiento desde lo disciplinar para la incursión al medio. Cabe mencionar que Colombia tenía, hasta el momento, cuarenta años de atraso en la implementación industrial, esto acompañado de las guerras civiles en las que el país se vio involucrado varias décadas, posterior a otras épocas y formas de violencia y guerra, en las que se destinaron vidas y recursos que podían haber sido aprovechados para la modernización industrial y el desarrollo.

México y Argentina, desde las políticas públicas de diseño, han apoyado procesos de crecimiento económico. En Colombia el ímpetu omnipotente del diseño ha individualizado los actores, restándole significado a las necesidades comunes; el diseño sigue siendo construido para la visualización y la contemplación, sin trascendencia alguna en los espacios públicos y proyectos colectivos para el desarrollo social (de Freitas, Chamy, \& Dumith, 2017).

Es necesario incluir en el Plan Nacional de Desarrollo un ramo específico para el diseño; que proteja, fomente y desarrolle la creación y el comercio de productos, ya que no existe difusión, claridad en la legislatura en materia de propiedad intelectual o industrial, facilidades para los autores, ni certidumbre en que las instancias de protección cuiden los derechos e intereses de creadores de innovación en el diseño (Zárate, Aguilar, \& Dupont, 2008, p. 143).

Recopilar las historias heroicas de diseñadores fundadores de facultades es un acto permanente. Mientras los diseñadores emprendedores sigan haciendo magia en microempresas y fabriquen números mínimos de series, con una tecnología obsoleta o rudimentaria, continuarán siendo titanes del diseño sin reseñar. Resulta interesante ver cómo el diseño en Colombia surge desde lo económico y lo histórico como una necesidad de las clases sociales para diferenciarse del morador común.

En países como Finlandia, Estados Unidos, Alemania, Austria, Japón y Suiza se establece el diseño como un factor diferencial de apoyo a las pymes, al desarrollo, la economía, a las personas, sociedades y culturas. En México se impulsan acciones articuladas entre sectores como la artesanía tradicional, el gobierno, los servicios y la alta tecnología, para que desde las leyes el diseño sea un factor diferencial y de prosperidad para las naciones. De todas maneras, el tema aún no ha sido comprendido desde su real importancia en el espacio latinoamericano. "En la carrera del progreso económico, parece que se pudiera despreciar la actividad social y cultural, el impacto ecológico y los efectos a largo plazo" (Papanek, 1977, p. 33).

Respecto a la educación tradicional del diseño, el currículo y plan de estudios como aporte de las academias regula las condiciones 
educativas y espacios críticos del universo en estudio, incluyendo la finalidad de acreditación de alta calidad y los componentes de autoevaluación y regulación de las instituciones de educación superior. Regido por necesidades del medio, que suscitan divergencias críticas sobre las condiciones iniciales y prospectivas del diseño industrial, las universidades latinoamericanas con 53 programas de diseño hasta 1992, adoptan posturas que aspiran al desarrollo de diseñadores industriales en países con industrialización baja, ambigua y no permanente, con necesidades sentidas desde lo político más que desde lo industrial, lo que dificulta constantemente esa interconexión, lo que dificulta el reconocimiento en espacios laborales y profesionales, marginando en ocasiones la disciplina. Como sucede en otras disciplinas afines, el profesional encuentra en la academia una oportunidad laboral, con retos diversos según la claridad de los perfiles profesionales, lo cual marca permanentemente el ritmo de las instituciones, involucrando temas de currículo como el ethos oculto, influenciador, permanente y silencioso del conocimiento.

Estamos infectados por la fragilidad de un presente que demanda cimientos firmes donde no existe ninguno [...]. Mientras contemplamos los cambios, nos encontramos constantemente desgarrados entre el deseo y el miedo, entre la anticipación y la incertidumbre (Bauman, 2013, p. 28).

A pesar de los esfuerzos para la implementación de aspectos más sostenibles y reales, las escuelas de diseño se mantienen al margen de una incorporación formal de la sostenibilidad. Los intereses económicos ligados a la productividad apartan las temáticas ambientales como diferencial en la formación de diseñadores, al no encontrar un área digna del desempeño (Cui, Fang, \& Zhou, 2018; Trevino Sherk \& Cobreros Rodriguez, 2019).

\section{Desde las academias y para los diseñadores}

Histórica y socialmente, el mercado ubica al profesional en diseño en espacios laborales de operación de software, ilustración o la publicidad. El diseñador dignifica esos espacios a pesar de no tener claras sus funciones y entender de forma integral la potencialidad en campos como la innovación, la sustentabilidad o los bio-materiales; su labor se reduce a la reproducción de elementos efímeros y de contemplación, carentes de valor y aporte social, que con el desarrollo necesario pueden potencializarse y hacer crecer las economías de escala.

Posterior a su formación, el diseñador reconoce sus competencias y las complementa con estudios técnicos o especializaciones de los que hace lectura permanente en las ofertas de empleo y del medio, lo cual le permite interiorizar unas expectativas enfocadas a lo operativo, olvidando lo proyectual, la investigación, la indagación y el cambio.

La búsqueda del pensamiento general y los consensos disciplinares tiene caminos espinosos; esto realmente afecta la sostenibilidad del diseño y el diseño para la sostenibilidad. Los esfuerzos ambientales siguen siendo mínimos. Es común ver muchos diseñadores que no 
interiorizan como mínimo el ACV (Análisis del Ciclo de Vida), para acompañar los procesos actuales y futuros de formación (Khan et al., 2017). Es necesario hacer una revolución hacia las realidades y caminos más eficaces del diseño. Esto no surge solo desde la empatía personal en la temática, sino desde la necesidad personal y profesional de verse en áreas en las que se sienta más afinidad y mayor proyección. "El marco en el cual debe moverse el diseño actual tiene que ser más social que productivo, más resolutivo que creativo y especulativo, más idealista que escenográfico" (Viñolas, 2005, p. 201). Estando presente la des agremiación disciplinar y la tendencia de pensamiento divergente, construida por cada escuela, los espacios académicos se convierten en áreas con matices de contradicción. La literatura formal en diseño en Colombia es muy difusa, y aunque el ejercicio de los teóricos e investigadores es permanente, la presión académica resultadista dificulta la verificación y construcción de documentos de calidad que fortalezcan un pensamiento autónomo, crítico y unificador del diseño. La formación de docentes en diseño corresponde a diversas áreas con o sin afinidad disciplinar, permanentemente enfocadas a carencias del pregrado como la administración y el mercadeo, donde se presentan oportunidades laborales más competitivas; acompañado de esto, algunos docentes se abstraen de espacios laborales comunes para los profesionales del diseño, en donde las actividades distan sustancialmente de las propuestas en el aula y se dificulta la vinculación laboral y personal al medio profesional.

La monetización de la academia dificulta permanentemente el pensamiento real o crítico. La sobrecarga laboral acompaña a los docentes, que obtienen plazas en diversas instituciones, lo que dificulta el crecimiento intelectual, profesional y personal, que trasciende a los estudiantes, quienes perciben como desinterés el efecto del burnout o síndrome del quemado que sufre el docente en Colombia. La rotación laboral permanente, las contrataciones no competitivas y la desconexión de la academia y el medio real o industrial desequilibra las oportunidades de construcción de un conocimiento compuesto, que ubique desempeños acertados y actuales, incluyendo la sostenibilidad. Los espacios aportan considerablemente en la consecución de un trabajo operativo y en ocasiones mal remunerado, debido al desconocimiento del quehacer en el medio, al bajo fortalecimiento de los nuevos conocimientos en diseño. Por ello, se deben asumir las responsabilidades académicas y éticas relacionadas con la formación y el deber como actor social. Directrices como la interdisciplinariedad, el fortalecimiento del trabajo en equipo, las comunidades globales y las habilidades personales pueden apoyar la renovación curricular para la formación de una nueva generación de diseñadores.

Las generaciones actuales de estudiantes no se alimentan de lo tradicional; esto debe ser claro para el docente, junto a una política seria de las facultades o instituciones. Educar para el siglo XXI es un reto a abordar en la inmediatez; la flexibilidad de los contenidos y espacios debe permitir la exclusión o inclusión de componentes, que redirijan el conocimiento 
hacia desarrollos específicos de competencias y habilidades personales y de contexto (França, Broman, Robèrt, Basile, \& Trygg, 2017).

"El plan de estudios sería una malla elástica, aquellas actividades y técnicas que exigen la resolución creativa de problemas. No puede darse separación entre el trabajo y las actividades de tiempo libre" (Papanek, 1977, p. 325).

Como consecuencia, la disciplina carece de la fortaleza sistémica que respaldaría los procesos de apoyo desde el diseño a la economía, su finalidad inicial. Los currículos de diseño en algunas instituciones carecen de herramientas administrativas o que enfoquen al estudiante hacia la especialización (Perpignan, Robin, \& Girard, 2017).

\section{Hallazgos para diseñadores}

Disciplinariamente hay coincidencias entre el ámbito nacional y el internacional, con diferencias marcadas en el pensamiento contextual colombiano. Algunas propuestas curriculares se orientan a mayor flexibilización de las materias y cómo se puede, desde el proceso formativo, trabajar en lo que quieren los estudiantes. Las camisas de fuerza en la educación son espacios moribundos de la pedagogía.

Es importante reconocer el esfuerzo de las academias en Colombia por incluir en sus planes de estudio y proyectos educativos de programa, o en los perfiles ocupacionales, algunas áreas asociadas al desarrollo sostenible; aun así, es evidente que no existe un eje transversal asociado a la especificidad en esta temática que apuntala esfuerzos desde lo global. De forma contradictoria se impulsa la formación al ciudadano global desde el espacio de formación.

Por ello el diseño debe comprender que los estudiantes de hoy no son los de ayer, tampoco serán los del mañana, "como era en un principio". La velocidad en la que la información circula será superada por el tiempo para estar informado y la educación deberá mutar hasta encontrar ese espacio límite en el que se genera conocimiento más profundo. "Habrá que evaluar desde la sociología si queremos ese conocimiento".

Como el saber disponible se duplica ya cada siete años, y en el 2030 se duplicará cada 72 días, el tiempo necesario para mantenerse informado, aprender, llegar a ser y mantenerse empleable se incrementará a la par. Lo mismo ocurrirá con el tiempo para cuidarse y entretenerse. No cambiará, sin embargo, el tiempo necesario para dormir o amar. (Atalli, 2006, p. 138)

A las escuelas no parece interesarles deconstruir el diseño, desaprender requiere una energía que puede estar mejor enfocada en atraer estudiantes, hacer que permanezcan cinco años en la academia y que, con suerte, reconozcan en el medio las habilidades no obtenidas en el espacio educativo. Los planes de estudio parecen estar proyectados con los escombros de las reconstrucciones, "hay materias y temáticas que no se adhieren ni con pegante". Entonces debe ser responsabilidad de las academias:

- Entender y entenderse desde el contexto nacional y/o global. 
Reconocer quiénes son sus estudiantes y por qué razones están allí.

Buscar espacios de co-creación para el conocimiento.

Unificar temáticas disciplinares que en el futuro permitan el reconocimiento profesional y cimienten bases epistemológicas de un diseño industrial colombiano.

La apuesta debe ser sostenible e innovadora en espacios desligados de los registros calificados o reconocimientos de alta calidad.

Generar y comunicar lineamientos claros a docentes y estudiantes de lo que van a aprender, les van a enseñar y les van a evaluar.

Aunar esfuerzos por encontrar la responsabilidad social de la educación, que debería ser un factor obligatorio del desarrollo y la educación.

Entender la diversidad ideológica, pero construyendo según la experticia de cada miembro de la comunidad académica, respondiendo al interrogante: ¿Cómo el diseño puede aportar en Colombia al desarrollo sostenible?

\section{Educación para el futuro}

La educación desarrollada en los siglos XVIII y XIX, bajo condiciones públicas no privadas, dificultaba la masificación y la transición con un rasero excluyente y poco real, sin lectura de las capacidades de las personas, lo cual no dista mucho de la calidad educativa de hoy en algunos contextos. La formación de ciudadanos con un conocimiento sectorizaba a las personas en dos estadios: económicos o intelectuales. Sir Ken Robinson formuló estas condiciones en libros, videos y textos, donde propuso el cambio de paradigmas y la ruptura de esquemas. Tanto el diseño como la educación actual deben ser DISRUPTIVAS. Allí surge la oportunidad disfrazada de dificultad.

Es pertinente mencionar que somos diferentes, diversos, y la transculturización ha aportado a los sistemas y métodos educativos permanentemente. Las universidades y sus actores se ven enfrentados a los cambios permanentes, en palabras de González (2008):

En las sociedades del conocimiento tendremos que aprender a desenvolvernos con soltura, en medio de la avalancha aplastante de informaciones, y también a desarrollar el espíritu crítico y las capacidades cognitivas suficientes para diferenciar la información útil de la que no lo es. (p. 3)

Por su lado, Becker (2017), en el informe de educación para el futuro, habla de los siguientes tópicos que generarán impacto en la educación. Es vital que los diseñadores y docentes entiendan que la era del conocimiento y la información tiene una velocidad diferente y más rauda cada vez, lo que obliga a los proponentes a actualizar en los cuerpos colegiados, empresarios, egresados y estudiantes las dinámicas de interés para el futuro. 
Se registra el siguiente consolidado de los retos actuales para la formación de profesionales para el futuro:

A largo plazo

- Promover la cultura de la innovación: la creatividad y el diseño como fuente de soluciones innovadoras para los problemas sociales del contexto.

- Aprendizaje profundo: "Saber cómo" la fórmula descrita es: pensamiento crítico + resolución de problemas + colaboración + aprendizaje personalizado $=$ alquimia educativa.

A medio plazo (para 2 o 3 años en adelante)

- Mayor enfoque en las métricas de aprendizaje: técnicas de análisis de aprendizaje y minería de datos que registran las interacciones de los estudiantes con los entornos digitales, sus progresos y su estado, se hace detección de los problemas y sus causas.

- Rediseñando los espacios de aprendizaje: los avances en tecnologías multimedia e inalámbricas, crear con ellas espacios más "inteligentes", motivadores y que permitan un aprendizaje más interactivo, colaborativo y motivador.

A corto plazo (de ahora en 1 año)

- Diseños de aprendizaje mixtos: se consolida como tendencia en todas las etapas de aprendizaje. La mezcla de los entornos tradicionales con nuevos entornos digitales ya sea en dispositivos móviles, en vivo o en la nube, parece que está siendo adoptada por todo el universo educativo.

- Aprendizaje colaborativo: colaborar para enseñar/aprender, atendiendo a los cuatro principios que propone el informe: poner al estudiante como centro, potenciar la comunicación y la interacción, trabajar en grupos, y resolver o diseñar soluciones para situaciones y retos reales.

De hoy en un año o menos:

- Tecnologías de aprendizaje adaptativo: aquellas que se adaptarán a la interacción del estudiante, a su progreso, y le ofrecerán el contenido que necesita para garantizar un progreso constante.

- Mobile learning: [...] la enseñanza y el aprendizaje se han hecho portables. El lugar y el dispositivo no son ya excusa para acceder a aplicaciones educativas de todo tipo (Icfpeuskadi, 2017, párr. 15).

En 2 o 3 años se verá más de cerca el internet de las cosas, la realidad aumentada, los sistemas de gestión del aprendizaje enfocados a la administración y personalización de contenidos, por lo que la inteligencia artificial y el uso de interfaces más intuitivas son retos del futuro para el diseñador y para las academias. 
Permitir una educación cooperativa lleva al estudiante a conocer unas necesidades específicas que pueden ser aplicadas en un entorno real, los cambios tecnológicos y sociales que los incitan a comprender cómo se debe pensar el mundo y qué saberes aplicados debe tener el profesional actual para que logre adaptarse al medio y dignificar su vida y su profesión. Dentro de las nuevas competencias se incluyen los valores, el desarrollo social y sostenible, apoyado obviamente por las nuevas tecnologías. Es posible establecer unas nuevas competencias que permitan la significación disciplinar y laboral así:

- Desarrollo de tecnologías generadoras de cambios socio culturales.

- Capacidad ambiental de producir diseños sustentables.

. Construcción de proyectos que impulsen la innovación social y el desarrollo económico.

- Control del ACV (Análisis de ciclo de vida) para avanzar a una sociedad más sostenible.

- Influir en la estética y signo del producto para un consumo más inteligente y responsable.

- Capacitar para posibilitar la réplica de los conocimientos en desarrollo sustentable.

Es oportuno que el diseñador cambie la formación tradicional de diseño de producto, debe expandir las aplicaciones y conocimientos a trabajos interdisciplinares más humanos que superen los límites de lo que proponía el siglo XX para su desempeño. Zachary Jean Paradis contestó a Bruce Nussman, autor del libro Inteligencia creativa, algunos conceptos que pueden guiar la estructura del nuevo diseño:

- El gran problema del diseño es la flexibilidad.

- Es como si se pusiera el vestido de acuerdo con el tema.

- Todos los métodos de diseño son sacados de otras disciplinas.

- Lo que la gente necesita, en eso se convierte el diseño.

- Es necesaria la especialización de las disciplinas.

- La sustentabilidad no es responsabilidad única de una disciplina, sino de todas.

\section{Educación para el desarrollo sostenible (EDS)}

Es clara la visión de la UNESCO para el año 2015, en su educación para todos y la educación para el desarrollo sostenible EDS. Confluyen allí aspectos de holística, transformación e inclusión. Resulta importante aclarar que muchos de los factores decisivos en el proyecto de la UNESCO y en el proyecto a presentar como fruto de la presente tesis parten de la idea de que la educación debe ser un proyecto global, y un proyecto acompañado por los diversos actores del proceso, los gobiernos, las asociaciones, los docentes y los estudiantes. En ese contexto, la universalidad juega un papel de primer orden para la agenda de educación del próximo decenio. "Incorporar las prácticas de la sostenibilidad en los 
contextos de educación y capacitación (con estrategias que abarquen al conjunto de las instituciones)" (Tang, 2014, párr. 8).

La UNESCO (2014) reconoció que es imposible cumplir las metas de alfabetización en el mundo; 774 millones de personas son analfabetas y las dos terceras partes son mujeres. Estas serán personas sin acceso a la educación superior y el sistema social-económico los excluirá; adicionalmente se reconocen las necesidades de las personas de establecer la equidad y la igualdad de género, y otras competencias que les permitan ingresar en nuevas economías y sociedades para mejorar su calidad de vida y la de sus familias.

La educación es la vía para erradicar la pobreza y la desigualdad, y debe ser un derecho para todos los habitantes del planeta, un principio de la sostenibilidad. Las mejoras en la educación deben darse con unos indicadores mundiales que permitan evaluar los estados y deficiencias de la educación, así como cuantificar el desarrollo para que este sea global y permita acoger a la mayor cantidad de personas. Las metas a proponerse en el año 2015 y que deben lograrse para 2030 deben estar dentro de los siguientes lineamientos generales:

- Inclusión del concepto de equidad de género.

- Alfabetización y básica primaria para niños y niñas, gratuita y obligatoria.

- Mayor inversión del PIB en educación.

- Formación en competencias globales y ciudadanos del mundo.

- Formación de docentes en competencias de sustentabilidad y globalidad.

- Trabajo digno como derecho a una vida más digna.

- Educación de calidad para todos.

Es importante trabajar en dos componentes que fundamentan el proyecto de educación en diseño: las habilidades y la formación docente. Esto hace parte de las políticas mundiales a implementarse, por ello Colombia como economía en desarrollo debe empoderar dichos retos para un crecimiento conjunto que permita el fortalecimiento social y económico.

Instituciones educativas comprometidas aportarían a la construcción de competencias básicas alrededor de las siguientes contribuciones de las disciplinas humanas:

- Matemáticas ayuda a los estudiantes a entender números extremadamente pequeños.

- Las artes del lenguaje, especialmente la alfabetización mediática.

- La historia enseña el concepto de cambio global, mientras ayuda a los estudiantes a reconocer el cambio que ha ocurrido durante siglos.

- La lectura desarrolla la capacidad de distinguir entre hechos y opiniones, y ayuda a los estudiantes a convertirse en lectores críticos. 
- Los estudios sociales ayudan a los estudiantes a comprender el etnocentrismo, el racismo y la inequidad de género, así como reconocer cómo se expresan en la comunidad y las naciones de todo el mundo.

La educación para el desarrollo sostenible busca la participación permanente del estudiante en las actividades de clase, fomentando las habilidades del pensamiento de nivel superior; entiende el entorno local, pero tiene la capacidad de adaptarse a cada cultura. Sugerido por la EDS, este modelo incluye el trabajo por fortalezas que contempla factores como la interdisciplinariedad y la identificación de los problemas asociados a la sostenibilidad.

En consecuencia, transversalizar el desarrollo sostenible, logrando la integración en todas las actividades académicas incluyendo el currículo, los contenidos, los modelos, la experiencia y la evaluación, hace parte de la formación integral que deben recibir los estudiantes de diseño. Los ejes transversales se convierten en instrumentos generalizados que permean toda la estructura educativa y están integrados en áreas del saber y el convivir. Estos ejes transversales son inter y transdisciplinares, derrocan el absolutismo del conocimiento de algunos catedráticos y se complementan mediante actividades de apoyo en el aula con simulaciones reales.

La incorporación de áreas como el ecodiseño, la gestión y la prospectiva son fundamentales para la academia; a su vez, esto beneficiará los actuales modelos de producción y consumo imprescindibles para la implementación del diseño para la sostenibilidad. Los "consumidores verdes" deben ser atendidos, las industrias exigirán estas características para cumplir sus metas de ventas, competir y mantenerse en el mercado bajo condiciones económicas sostenibles.

\section{Referencias}

Ahmad, S., Wong, K., Tseng, M., \& Wong, W. (2018). Sustainable product design and development: A review of tools, applications and research prospects. Resources, Conservation and Recycling, 132, 49-61. https://d oi.org/10.1016/j.resconrec.2018.01.020

ArtStudio Magazine. (2003). Bauhaus - Cuna del Arte Mundial. Obtenido de Especiales: http://www.artstudiomagazine.com/especiales/bauhaus.html

Atalli, J. (2006). Breve historia del Futuro. Barcelona: Paidós.

Baudrillard, J. (1968). El sistema de los objetos. Madrid: Siglo veintiuno.

Bauman, Z. (2007). Vida de Consumo. México, D.F.: Fondo de Cultura Económica.

Bauman, Z. (2013). Sobre la educación en un mundo liquido, conversaciones con Ricardo Mazzeo. Barcelona: Paidós.

Becerra, M. (2007). Formacion en competencias para el trabajo interdisciplinario del diseñador (Vol. 4).

Becker S., Cummins, M., Freeman, D., Hall, C., \& Ananthanarayanan, V. (2017). NMC Horizon report 2017 higher education edition. Texas, Austin: The New Media Consortium. 
Bolshakov, B., Shamaeva, E., \& Popov, E. (2019). E-learning system of knowledge engineering for design and management of sustainable innovative development of regional facilities. 2019 International Science and Technology Conference "EastConf", EastConf 2019. https://doi.o rg/10.1109/Eastonf.2019.8725409

Brawer, W. (2005). Seed Money (Vol. 4). Print.

Brezet, H., \& van Hemel, C. (1997). Ecodesign: A promising Approach to Sustainable Production and Consumption. París: United Nations Environment Programme.

BVSDE Desarrollo Sostenible . (2014). Manual de educación para el desarrollo sostenible - capítulo III reorientar la educación. Recuperado de: http://w ww.bvsde.paho.org/bvsacd/cd61/manualEDS/part1.pdf

Cámara de Diputados México. (2008). Memorias del foro por una política de diseño en México. México D.F.

CEPAL. (2014). Carbon Footprint and International Trade of Latin America and the Caribbean. Recuperado de Infographics: http://www.cepal.org/e $\mathrm{n}$ /infografias/la-huella-de-carbono-y-el-comercio

Conference, U., \& Development, S. (2009). Bonn Declaration.

Congreso de la República de Colombia. (2012). Ley 1549. Diario Oficial No. 48482 de julio 5 de 2012. Por medio de la cual se fortalece la institucionalización de la política nacional de educación ambiental y su incorporación efectiva en el desarrollo territorial. Bogotá.

Cortés, D., Cruz, A., Jani, G., \& Peréz, M. (2014). Imported design ideas and its spreading in Latin America: a historiographical critique. Fevereiro. 1(1), 141-144.

Cortés, D., Cruz, A., Jani, G., \& Peréz, M. (2014). Imported design ideas and its spreading in Latin America: a historiographical critique.

Cui, Y., Fang, X., \& Zhou, H. (2018). Green Design and Sustainable Development of School Uniforms. In W. K. (Ed.), 5th Annual International Conference on Material Science and Environmental Engineering, MSEE 2017 (Vol. 301). https://doi.org/10.1088/1757-899 $\mathrm{X} / 301 / 1 / 012122$

Chambouleyron, M., \& Pattini, A. (2004). El diseño y el imperativo ecológico. (spanish). Huellas búsquedas en artes y diseño. (Vol. 4). Mendoza: Universidad Nacional de Cuyo.

Davies, C. (2008). Learning and teaching in laboratories: an Engineering Subject Centre guide. Loughborough: Heigher Education Academy Engineering Subject Centre.

Design Schools Index. (s.f.). Top 10 Mejores Escuelas de Diseño Industrial en el mundo. Recuperado de Recent Post: http://www.designschoolsindex.c om/top-10-best-industrial-design-schools-in-the-world/

Dorst, C. (2006). Design Problems and Design Paradoxes Kees Dorst (Vol. 22).

Dorst, K., \& Sidney, T. (s.f.). The Nature of Design Thinking.

EcoLabels. (s.f.). Eco etiquetas. Recuperado de http://2.bp.blogspot.com/-PLD SpWG0O94/VgMGSWfP24I/AAAAAAAAAPs/a3oiQ4RFdGM/s16 00/eco-labels.jpg

Findeli , A. (2001). Rethinking Design Education for the 21st Century: Theoretical, Methodological, and Ethical Discussion (Vol. 1). Design Issues. 
Foro Económico Mundial. (2016). Future Jobs.

Galán, C. (2017). ¿Qué universidades ofrecen Diseño Industrial en Colombia? Recuperado de Blog Diseño Industrial, Estilo de Vida: http://printcolom bino.4mg.com/diseoindustrialcolombiaprogramas.htm

González, A. (2001). Ecodiseño, ingeniería de diseño de producto y los retos del Mercado Verde. Medellín: Universidad EAFIT.

González, J. (2008). TIC y la transformación de la práctica educativa en el contexto de las sociedades del conocimiento. Revista de Universidad y Sociedad del Conocimiento. 5(2), 1-8.

Hernández, R., \& Fernández, C. (2010). Investigación. México, D.F.: Mc Graw Hill.

Hernández, R., Fernández, C., \& Baptista, P. (2010). Metodología de la Investigación. México, D.F.: McGraw Hill.

Howarth, G., \& Hadfield, M. (2006). A sustainable product design model. Materials and Design. (27), 1128-1133.

ITD. (2015). Objetivos del Desarrollo Sostenible. Recuperado de http://www. itd.upm.es/wp-content/uploads/2015/10/ODS_SDG_vertical_baja.jpg

Juez, M. (2002). Contribuciones para una antropología del diseño. Barcelona: Gedisa Editorial.

Lai, Y., \& Peng, L. (2020). Effective teaching and activities of excellent teachers for the sustainable development of higher design education. Sustainability (Switzerland), 12(1). https://doi.org/10.3390/su12010028

Li, W., Ho, M., \& Yang, C. (2019). A design thinking-based study of the prospect of the sustainable development of traditional handicrafts. Sustainability (Switzerland), 11(18). https://doi.org/10.3390/su111848 23

Lilley, D., \& Lofthouse, V. (2009). Sustainable design education considering design for behavioural change. Engineering Education. 4(1), 4, 29-41.

Khan, M., Raoufi, K., Park, K., Reza, T., Psenka, C., Jackson, K., ... Kim, K. (2017). Development of learning modules for sustainable life cycle product design: A constructionist approach. 124th ASEE Annual Conference and Exposition, 2017-June. Recuperado de: https://www.scopus.com/inward/record.uri?eid=2-s2.0-850305556 08\&partnerID $=40 \& \mathrm{md} 5=5$ e26fe $7 \mathrm{fd} 2 \mathrm{~b} 94325 \mathrm{dee} 2363 \mathrm{cb} 831 \mathrm{dd} 96$

Kinokuni, H., Ohori, S., \& Tomoda, Y. (2019). Optimal Waste Disposal Fees When Product Durability is Endogenous: Accounting for Planned Obsolescence. Environmental and Resource Economics, 73(1), 33-50. h ttps://doi.org/10.1007/s10640-018-0248-6

Manzzini, E., \& Vezzoli. (2003). A strategic design approach to develop sustainable product service systems: examples taken from the "environmentally friendly innovation" Italian prize. Journal of Cleaner Production. 11(8), 11, 851-857.

Martín, M., Enrique, L., Silva, B., \& Arturo, F. (2012). Las competencias del diseñador industrial en el ámbito manufacturero en Ciudad Juárez (Vol. 21). México: Sección Monográfica.

Malik, K., \& Mikolajczak, E. (2019). Senior housing universal design as a development factor of sustainable-oriented economy. Sustainability (Switzerland), 11(24). https://doi.org/10.3390/su11247093

McDonough, W. (2005). Cradle to Cradle. Madrid: McGraw-Hill . 
Montgomery, D. (2007). Ecostyle (Vol. 29). In Business.

Naciones Unidas. (2015). Transformar nuestro mundo: la Agenda 2030 para el Desarrollo Sostenible. Recuperado de Iniciativa Interagenciales: http://hn.one.un.org/content/unct/honduras/es/home /objetivos-del-milenio-para-el-desarrollo.html

Nichols, B. (2013). Valuing the Art of Industrial Design. Washington, D.C. : National Endowment for the Arts .

Niño, L. (Enero de 2015). Obsolescencia programada como fenómeno cultural. MasD, Revista Digital de Diseño, 9(16), 104-109.

Pacey, E. (2008). A change is gonna come (Vol. 23). Design Week.

Papanek, V. (1977). Diseñar para el mundo real: ecología humana y cambio social. Barcelona: Pol-len.

Paricio, J., Peña, J., \& Miralbes, R. (2019). Well planned obsolescence and the eco-design. Lecture Notes in Mechanical Engineering, 74-84. https://do i.org/10.1007/978-3-030-12346-8_8

Perpignan, C., Robin, V., \& Girard, P. (2017). French education system organization from secondary school to university to prepare future engineers to sustainable development and eco-design (C. A. \& C. D., Eds.). 6th International Conference on Research into Design, ICoRD 2017, Vol. 66, pp. 373-384. https://doi.org/10.1007/978-981-10-3521-0_32

Programa de Naciones Unidas para el Medio Ambiente. (2010). Aquí y ahora. Educación para el desarrollo sostenible, recomendaciones y orientaciones. Paris: UNEP.

Quacquarelli Symonds. (2017). World University Rankings by subjects. Recuperado de https://www.topuniversities.com/subject-rankings/2017

Real Academia Española. (s.f.a). Diseño. Recuperado de Diccionario de la lengua española: http://dle.rae.es/srv/search?m $=30 \& w=$ dise\%C3\%B1o

Real Academia Española. (s.f.b). Sostenible. Recuperado de Diccionario de la lengua española: http://dle.rae.es/?id=YSE9w6H

RES. (2012). Los 10 mandamientos del diseño sostenible. Recuperado de Diseño: https://www.ecointeligencia.com/2012/11/10-mandamientosdiseno-sostenible/

Rizo, S. (2004). Ecodiseño. Ingenieria del ciclo de vida para el desarrollo de productos sostenibles. Valencia: Alfaomega.

Reuter, I., \& Reiter, S. (2019). Development of a multi criteria analysis method to optimize the sustainable architectural design of residential buildings. In B. U. \& A. F. (Eds.), 10th International Conference on Indoor Air Quality, Ventilation and Energy Conservation in Buildings, IAQVEC 2019 (Vol. 609). https://doi.org/10.1088/1757-899X/609/7/072062

Ruth-Trumpold. (s.f.). Clean Technology. Recuperado de Resource management and sustainable production: http://www.ruthtrumpold.id.a u/destech/?page_id $=1474$

Salgado, C., \& Trellez, E. (2009). Políticas, estrategias y planes regionales, subregionales y nacionales en educación para el desarrollo sostenible y la educación ambiental en América Latina y el Caribe. Decenio de las Naciones Unidas de la Educación para el Desarrollo Sostenible 2005-2015.

Salinas, O. (1992). Historia del Diseño Industrial. México: Trillas. 
Sam. (2015). Y cuando ya no sirvas más: obsolescencia planificada. Recuperado de Archivo: http://hometech.com.mx/y-cuando-ya-no-sirvas-mas-obsole scencia-planificada/

Tang, Q. (2014). Aprender hoy para un futuro sostenible. Recuperado de Educación: http://www.unesco.org/new/es/education/resources/online -materials/single-view/news/learning_today_for_a_sustainable_future/

Teng, T., \& Qu, C. (2018). Urban landscape design based on sustainable development innovation. Open House International, 43(1), 68-72. Recuperado de: https://www.scopus.com/inward/record.uri?eid=2-s2.0-850451225 66\&partnerID $=40 \& \mathrm{md} 5=526 \mathrm{a} 14663 \mathrm{e} 10045 \mathrm{acab} 14 \mathrm{ab} 712 \mathrm{~b} 669 \mathrm{eb}$

Trevino, J., \& Cobreros, C. (2019). Rural democratic design: Participatory design and service learning strategies in sustainable development to promote civic mindedness in community development. In B. E., K. A., B. L., B. R., E. D., G. H., ... W. R. (Eds.), 21st International Conference on Engineering and Product Design Education, E and PDE 2019. Recuperado de: https://www.scopus.com/inward/record.uri?eid=2-s2.0-850840217 61\&partnerID $=40 \& \mathrm{md} 5=8 \mathrm{~b} 40 \mathrm{fd} 4 \mathrm{a} 0 \mathrm{e} 6 \mathrm{~d} 71 \mathrm{e} 27477225 \mathrm{~d} 938 \mathrm{~d} 308 \mathrm{f}$

Tsitman, T., \& Proshunina, K. (2019). Ecological design based on biomodels in sustainable development. In P. M. \& M. V. (Eds.), 12th International Scientific Conference on Agricultural Machinery Industry, INTERAGROMASH 2019 (Vol. 403). https://doi.org/10.1088/1755$1315 / 403 / 1 / 012236$

Torkington, S. (2017). Los trabajos del futuro... y dos habilidades que necesita para obtenerlos. Recuperado de: Empleo y habilidades: https://www.weforum.org/es/agenda/2017/05/los-trabajos -del-futuro-y-dos-habilidades-que-necesita-para-obtenerlos/

UNESCO. (2009). Bonn Declaration. Bonn: World Heritage Committee.

UNESCO. (2012). Educación para el desarrollo sostenible. [Libro de Consulta]. París, Francia: UNESCO.

UNESCO. (2014). Reunión Mundial sobre la Educación para todos: Declaración final de la reunión mundial sobre la EPT de 2014 el acuerdo de mascate. Mascate: UNESCO.

UNESCO. (2017). La educación transforma vidas.

UNESCO. (s.f.). Teaching and Learning for a Sustainable Future. Recuperado de Education: http://www.unesco.org/education/tlsf/

Vallet, F., Eynard, B., \& Millet, D. (2014). Proposal of an Eco-design Framework based on a Design Education Perspective (Vol. 15). Procedia CIRP.

Viñolas, J. (2005). Diseño ecológico: hacia un diseño y una producción en armonía con la naturaleza. Barcelona: Blume.

Wiprächtiger, M., Haupt, M., Heeren, N., Waser, E., \& Hellweg, S. (2020). A framework for sustainable and circular system design: Development and application on thermal insulation materials. Resources, Conservation and Recycling, 154. https://doi.org/10.1016/j.resconrec.2019.104631

World Economic Forum. (2016). Future Jobs. Cologny: World Economic Forum.

Yi-Fei, G. (2017). Green Innovation Design of Products under the Perspective of Sustainable Development. 2016 International Conference 
on Environmental Engineering and Sustainable Development, CEESD 2016, 51(1). https://doi.org/10.1088/1742-6596/51/1/012011

Zárate, J., Aguilar, V., \& Dupont, M. (2008). Memorias del foro por una política de diseño en México. México, D.F.: Cámara de Diputados México. 